\title{
SOME EXAMPLES OF NONFINITE DIFFEOMORPHISM GROUPS
}

\author{
TERRY C. LAWSON
}

\begin{abstract}
An example due to Guy Hirsch is modified to give examples of manifolds $N$ such that Diff $N$ is not dominated by a finite complex.
\end{abstract}

In this note we will give some examples of compact differentiable manifolds $N$ such that Diff $N$ (with the $C^{\infty}$ topology) is not dominated by a finite complex. Our examples are all motivated by an example of Guy Hirsch [2] which shows that $\pi_{2}$ (Diff $S^{3} \times S^{1}$ ) is nonzero. For other results on nonfinite diffeomorphism groups see [1].

We now describe Hirsch's example. Let $\xi=\left(S^{7}, h, S^{4}\right)$ be the Hopf bundle. Let $f: S^{3} \times S^{1} \rightarrow S^{4}$ be a $C^{\infty}$ map of degree one and let $f^{*} \xi=$ $\left(E, \pi, S^{3} \times S^{1}\right)$ be the induced bundle. Consider the diagram

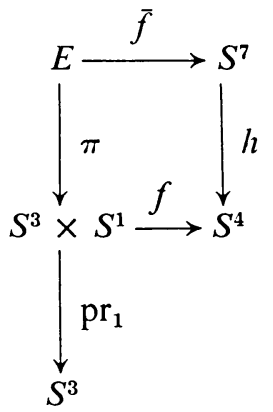

The composition $E \rightarrow{ }^{\pi} S^{3} \times S^{1} \rightarrow{ }^{\mathrm{pr}_{1}} S^{3}$ is a differentiable bundle map; the fiber is $S^{3} \times S^{1}$ and the structure group is Diff $S^{3} \times S^{1}$. Comparison of the Serre spectral sequences for $\pi$ and $h$ shows that $E$ does not have the homology of $S^{3} \times S^{3} \times S^{1}$. Hence the bundle $\eta=\left(E, \mathrm{pr}_{1} \circ \pi, S^{3}\right)$ is nontrivial. Consequently, its characteristic map is a nontrivial element of $\pi_{2}\left(\right.$ Diff $\left.S^{3} \times S^{1}\right)$.

This example was given by Hirsch in [2] to show that the existence of a cross section to a bundle does not imply that the additive cohomology is

Received by the editors October 8,1971 .

AMS 1969 subject classifications. Primary 5710, 5755.

Key words and phrases. Diffeomorphism group, finite complex, differentiable fiber bundle, structure group.

(c) American Mathematical Society 1972 
isomorphic to that of a product space, even if the fiber is a Lie group. Later, Serre (as Hirsch remembers it) pointed out to him that the structure group of the bundle could not be a Lie group. Thus the example disproved a conjecture that any differentiable fiber bundle may have its structure group reduced to a Lie group. It was communicated to me in that form by Hans Samelson.

We now modify the construction to prove:

THEOREM. Let $i, j$ be odd integers, $1 \leqq j \leqq i-2$. Let $M$ be any compact oriented differentiable manifold of dimension $j$. Then $\pi_{i-j}\left(\right.$ Diff $\left.M \times S^{i}\right)$ contains elements of infinite order.

Proof. Let $\xi=\left(E, \pi, S^{i+1}\right)$ be any orthogonal bundle over $S^{i+1}$ with fiber $S^{i}$ such that $E$ is a rational homology sphere. Many such bundles exist. If the characteristic map of $\xi$ is $\alpha \in \pi_{i}(S O(i+1))$, the condition for $E$ to be a rational homology sphere is that $p_{*} \alpha \neq 0 \in Z \approx \pi_{i}\left(S^{i}\right)$,

$$
p_{*}: \pi_{i}(S O(i+1)) \rightarrow \pi_{i}\left(S^{i}\right) .
$$

For the cell structure of $E$ is easily seen to be $S^{i} \cup_{p_{*}} e^{i+1} \cup e^{2 i+1}$ and so $E$ is a rational homology sphere if and only if $p_{*} \alpha \neq 0$. But for $i$ odd, $p_{*}$ is surjective onto $2 Z \subset Z \approx \pi_{i}\left(S^{i}\right)$; it is onto $Z$ for $i=1,3,7$. The map $p_{*}$ measures the obstruction to a cross section of $\xi$, i.e. $\left(p_{*} \alpha\right) \mu$ is the Euler class of the bundle $\xi$ where $\mu$ is the fundamental class. A choice of $\xi$ which works for all odd $i$ is the tangent bundle of $S^{i+1}$.

Let $f: M \times S^{i+1-j} \rightarrow S^{i+1}$ be a map which is of degree one. Let $\xi^{\prime}=$ $f * \xi=\left(E, \pi^{\prime}, M \times S^{i+1-j}\right)$ be the induced bundle. By examination of the induced map of Serre spectral sequences for $\xi$ and $\xi^{\prime}$, using the fact that $E$ is a rational homology sphere, we see that $E^{\prime}$ does not have the same rational homology as the product $M \times S^{i+1-j} \times S^{i}$. We form a new (differentiable, not orthogonal) bundle $\xi^{\prime \prime}$ from $\xi^{\prime}$ by composing the two bundle maps $E^{\prime} \rightarrow{ }^{\pi^{\prime}} M \times S^{i+1-j} \rightarrow{ }^{\mathrm{pr}_{2}} S^{i+1-j}$. The structural group of the bundle is Diff $M \times S^{i}$ and its characteristic map lies in $\pi_{i-j}\left(\right.$ Diff $\left.M \times S^{i}\right)$. Since $E^{\prime}$ does not have the rational homology of the product, its characteristic map is nonzero. Moreover, another application of the Serre spectral sequence shows that its characteristic map must have infinite order.

Corollary. Let $i, j, M$ be as in the theorem. Then Diff $M \times S^{i}$ is not dominated by a finite complex.

Proof. Diff $M \times S^{i}$ is a topological group. If it were dominated by a finite complex, then its even dimensional homotopy would be finite [4].

The corollary should be contrasted with the results of [1]. There examples are given of many manifolds whose diffeomorphism groups are not of finite homotopy type. The examples depend on the pairings of Milnor 
and Milnor-Munkres-Novikov and are very much dependent on being in the differentiable category-the elements constructed become zero when one passes to the $P L$ or topological categories. Our examples are of a much more general phenomenon. We could assume that $M$ is a topological manifold and prove that Homeo $M \times S^{i}$ is not dominated by a finite complex.

For the case where $M$ is itself a sphere we may give a different explanation for the phenomenon of the theorem. This is really "what is going on" for general $M$ also, since all we use of $M$ is a disk; but it is much more awkward to state in the general case. Recall that the characteristic map for the bundle we construct lies in $\pi_{i-j}\left(\right.$ Diff $\left.S^{i} \times S^{j}\right)$. Analogous to the Gromoll homomorphism there is a homomorphism $\pi_{p}\left(\right.$ Diff $\left.S^{r} \times S^{s}\right) \rightarrow$ $\pi_{0}\left(\right.$ Diff $\left.S^{r+p} \times S^{s}\right)$. One uses an adjoint construction and the inclusion $D^{p} \times S^{r} \subset S^{p+r}$. Moreover, this map factors as

$$
\begin{aligned}
\pi_{p}\left(\text { Diff } S^{r} \times S^{s}\right) & \rightarrow \pi_{p-1}\left(\text { Diff } S^{r+1} \times S^{s}\right) \\
& \rightarrow \cdots \rightarrow \pi_{p-j}\left(\text { Diff } S^{r+j} \times S^{s}\right) \rightarrow \cdots \rightarrow \pi_{0}\left(\text { Diff } S^{r+p} \times S^{s}\right) .
\end{aligned}
$$

Examination of the construction of $\xi^{\prime \prime}$ shows that its characteristic map is in the image of the characteristic map for $\xi$ in the factorization

$$
\pi_{i}(S O(i+1)) \subset \pi_{i}\left(\text { Diff } S^{i}\right) \rightarrow \pi_{i-j}\left(\text { Diff } S^{i} \times S^{j}\right) \rightarrow \pi_{0}\left(\text { Diff } S^{i} \times S^{i}\right) .
$$

But the total map is a monomorphism [3] and the characteristic map for $\xi$ has infinite order, so the characteristic map for $\xi^{\prime \prime}$ has infinite order.

\section{REFERENCES}

1. P. L. Antonelli, D. Burghelea and P. J. Kahn, The non-finite homotopy type of some diffeomorphism groups. I, Topology 11 (1972), 1-49.

2. G. Hirsch, Sur les groupes d'homologie des espaces fibrés, Bull. Soc. Math. Belg. 6 (1953), 79-96. MR 16, 1142.

3. J. Levine, Self-equivalence of $S^{n} \times S^{k}$, Trans. Amer. Math. Soc. 143 (1969), 523543. MR 40 \#2098.

4. J. P. Serre, Groupes d'homotopie et classes de groupes abéliens, Ann. of Math. (2) 58 (1953), 258-294. MR 15, 548.

Department of Mathematics, Tulane University, New Orleans, Louisiana 70118 